\title{
Innervation of the thoracolumbar fascia
}

\author{
Siegfried Mense \\ Department of Neurophysiology, Medical Faculty Mannheim, Heidelberg University, \\ Mannheim, Germany
}

This article is distributed under the terms of the Creative Commons Attribution Noncommercial License (CC BY-NC 4.0) which permits any noncommercial use, distribution, and reproduction in any medium, provided the original author(s) and source are credited.

\begin{abstract}
The aim of the study was to obtain information on the sensory functions of the thoracolumbar fascia (TLF). The types of nerve fibres present in the TLF were visualized with specific antibodies to neuropeptides and sympathetic fibres. Most data were obtained from the TLF in rats, but some findings from the human fascia are also included. The only receptive nerve ending found was the free nerve ending, i.e. no corpuscular receptors existed in our specimen. An exclusive innervation with free nerve endings speaks for a nociceptive function, but the TLF may also fulfill proprioceptive functions, since many of the free nerve endings have a low mechanical threshold. Most of the fibres could be visualized with antibodies to CGRP [calcitonin gene- related peptide (CGRP)] and SP [substance P (SP)]. The latter ones most likely were nociceptors. The TLF contained a great proportion of postganglionic sympathetic fibres, which may be vasoconstrictors. A comparison between an inflamed and intact fascia showed an increase of the CGRP- and SP-positive fibres in the inflamed TLF. This finding could be one explanation for the low back pain of many patients, since practically all lesions of the fascia are accompanied by a sterile inflammation.
\end{abstract}

Key Words: Thoracolumbar fascia, innervation, free nerve endings, proprioception, nociception, fasciitis.

Eur J Transl Myol 29 (3): 151-158, 2019

Presently, the official fascial nomenclature defines fascia as a three-dimensional network that includes all connective tissues that invest or interweave between inner organs and muscles. ${ }^{1}$ This usage of the term fascia has the advantage of simplifying communication, but the disadvantage is that all possible connective tissues with different histological composition are lumped together. Therefore, when the fascia of the low back is addressed, one has to specify if the subcutaneous tissue or the intervertebral discs are talked about. Actually, there can be great differences in the structure and function of fasciae depending on the body region. ${ }^{2,3}$ The present article deals with the thoracolumbar fascia $[(\mathrm{TLF})]$ in rats and humans. The emphasis will be on the innervation of the TLF. ${ }^{4}$ Nowadays, there can be no doubt that fasciae are innervated, but for many years the extent and nature of the innervation was a matter of discussion. For instance, Bednar and colleagues stated that in patients with non-specific low back pain the TLF was "deficiently innervated".5 The reason for this statement was that they found no sensory receptors in the specimen they had studied. On the other hand, the investigations by Yahia et al. (1992) had shown that the human TLF from healthy controls was innervated with free and corpuscular sensory endings. ${ }^{6}$ At present, the innervation of fasciae is an accepted fact, although differences between various fasciae exist with regard to the composition of the fibre spectrum and the density of innervation. The aim of the paper is to describe the sensory functions of the TLF. Mainly two functions are being discussed, namely proprioception and nociception.

\section{Materials and Methods}

The interpretations of the present report are largely based on immunohistological data from rats. All animal data were obtained from adult male Sprague Dawley rats. The experiments were carried out in accordance with the German law on the protection of animals and the ethical proposals of the International Association for the Study of Pain. ${ }^{7}$ In order to see if an experimental fasciitis influences the fibre density of the TLF, also data from inflamed TLF are included. In this article, the term "TLF" includes the subcutaneous tissue and the thoracolumbar fascia proper covering the multifidus muscle. As a universal marker for all neuronal structures antibodies to the neuron-specific protein PGP 


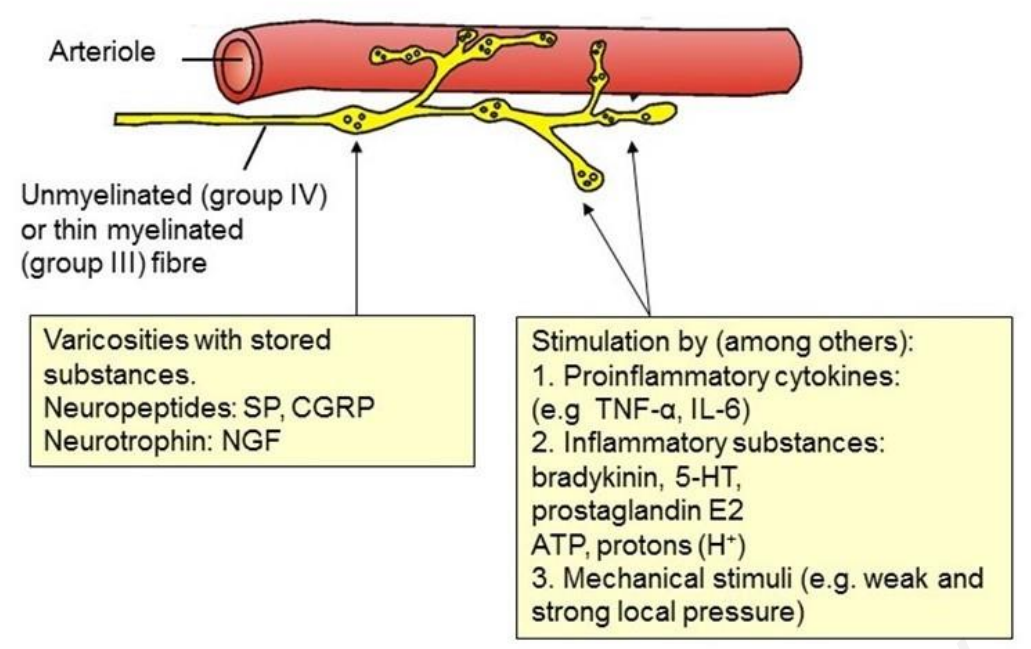

Fig 1. Schematic drawing of a free nerve ending innervating an arteriole. The ending exhibits several axonal widenings (varicosities) in which neuropeptides and neurotrophins are stored. When the ending is stimulated by chemical or mechanical stimuli, the contents of the varicosities are released and influence the nearby blood vessels leading to vasodilation and increase in capillary permeability. ATP and protons are particularly effective stimuli for endings in deep soft tissues. Please note that many free nerve endings have a low mechanical threshold and might function as proprioceptors.

9.5 [(protein gene product 9.5)] were used. Other antibodies were directed towards the neuropeptides CGRP [(calcitonin gene-related peptide)], SP [(substance P)] and the neurotrophin NGF [(nerve growth factor)] which all are present in the axoplasm of free nerve endings. Antibodies binding to TRPV1 [(transient receptor potential cation channel subfamily $\mathrm{V}$ member 1)] served to visualize the receptor molecule on nociceptors, and antibodies to $\mathrm{TH}$ [(tyrosine hydroxylase)] was used as a marker for postganglionic sympathetic fibres. The antibodies were purchased from the following companies: Bachem, Chemicon, Alomone Labs, Vector. Some of the fascia specimen were obtained from patients who had to undergo an operation of the spine because of an acute accident. Prior to the biopsies, informed consent was obtained from these patients. In the text, besides "nerve fibre" the term "free nerve ending" will be used. It denotes preterminal portions of the axon which exhibit at least 4 varicosities. Varicosities are axonal widenings that contain neuropeptides and the neurotrophin NGF. When the sensory ending is excited by a stimulus, it releases the substances stored in the varicosities (Figure 1). The free nerve ending with its varicosities is the site where a stimulus acts. The specimen were taken from the low back, close to the spinous process L4-L5 in the rat and L5 in humans. In addition to the subcutaneous tissue the TLF had 3 layers (Figure 2): A. Outer layer which consists of a thin sheet of parallel collagen fibers that are oriented perpendicularly to the spine. B. Middle layer which contains thick bundles of collagen fibres that run obliquely to the spine. C. Inner layer which consists of loose connective tissue. The inner layer was the only one that contained a few elastic fibres. The distribution of nerve fibres and endings was not uniform over the various layers. The middle layer was largely free of free endings with most of the fibres and endings being located in the superficial fascia (subcutaneous tissue and outer layer) as well as in the inner layer. Corpuscular endings were not found, neither in rat nor in human specimen. Since several authors had described these endings in the TLF, we tried several staining techniques but couldn't find any sensory corpuscles. All we could find in several hundred specimen were free nerve endings. One possible explanation for this discrepancy is that the regions of the TLF studied differed (our evaluation was restricted to a region $5 \mathrm{~mm}$ lateral to the spinous process in rats). Actually, in more lateral areas, in the transition zone to the flat abdominal muscles, we found some Golgi tendon organs. Generally, free nerve endings are assumed to be connected to the spinal cord by non-myelinated (group IV) or thin myelinated (group III) fibres. ${ }^{8}$ The free endings can be excited by chemical and mechanical stimuli (Figure 1). Not all of the endings are nociceptive, approximately $45 \%$ have a low mechanical threshold and can be activated by weak mechanical stimuli such as non-noxious local pressure ${ }^{9}$ The free nerve endings in the rat and human fascia looked exactly the same. All of them had the appearance of a string of pearls as shown in Figure 3, and many exhibited a chain of varicosities. In the figures of this article (Figures 4-6), all nerve fibres have a brown colour irrespective of their antigen content, because in 


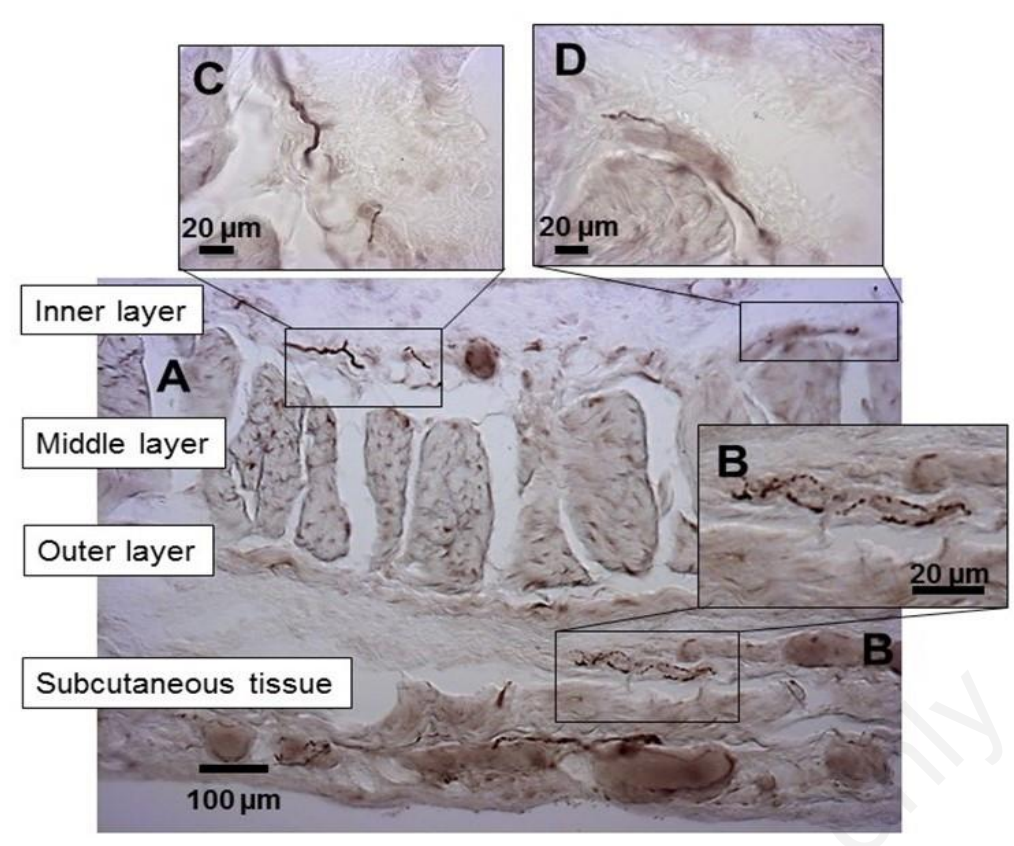

Fig 2. Cross-section through the rat thoracolumbar fascia with its three layers plus subcutaneous tissue (A). The section was stained with antibodies to PGP 9.5 to show all neuronal structures, irrespective of their function. $B$, free nerve ending with chains of varicosities. $C, D$, fibres of passage that terminate somewhere outside the section.

all cases the indirect immunohistochemical staining with 3,3-diaminobenzidine tetrahydrochloride [(DAB)] as a chromogen was employed. For quantitative evaluation of the fibre density we used cross sections of the rat fascia. As a measure of fibre density the length of all fibres and endings was added and is given as fibre length per $1000 \mu 2$ in Figure 7. The first section was determined randomly, and then every 5th section was evaluated (computer-assisted). This procedure reduces double-counting. Comparisons between control rats and animals with inflamed fascia were made using the Utest of Mann and Whitney. A probability level of less than 5\% (two-tailed) was regarded significant.

\section{Results}

\section{Fibres and endings stained with antibodies to PGP 9.5}

This stain was applied to obtain an overview of all neuronal elements that were present in a given histological section of the TLF. In addition to the fascia layers, Figure 2 shows examples PGP 9.5-stained fibres in the rat. Truncated fibres of passage that terminate somewhere outside the section are visible in the inner layer (insert $C ; D$ ) as well as in the subcutaneous tissue and outer layer. In the insert B, a free nerve ending is shown at higher magnification. The same fibres and endings were also present in the human TLF. The free nerve endings in these specimen looked identical to those found in the rat TLF, and many fibres were located in the adventitia of small arteries (Figure 3)
Neuropeptide-containing afferent fibers and endings (units)

CGRP-containing units

These units are partly mechanoreceptive and partly nociceptive. ${ }^{9,10}$ In our material from rat and human TLF, the CGRP-ir [(CGRP-immunoreactive)] fibres and endings were the most frequent ones. Figure 4 shows a whole mount preparation that contains branching CGRP-ir fibres with some varicosities in the lower left part of the figure. Apparently, the stem fibre to the right accompanies a blood vessel. In this figure, the focus of the microscope was adjusted so that predominantly structures in the subcutaneous tissue including some fat cells are visible.

\section{SP-containing units}

All units containing SP are assumed to be nociceptive. ${ }^{10,11}$ SP-containing nerve fibres and endings were rare when compared with CGRP-ir ones. The SP-ir units were completely absent from the middle layer of the fascia and were found mainly in the outer layer and the subcutaneous tissue. One possible reason for the absence of SP fibres in the middle layer is that this layer consisted of thick and densely packed collagen fibre bundles which are under mechanical stress when the trunk is bent. In this situation, the fiber bundles move against each other and could deform the SP-ir units. This could lead to an excitation of the ending. Figure 5 

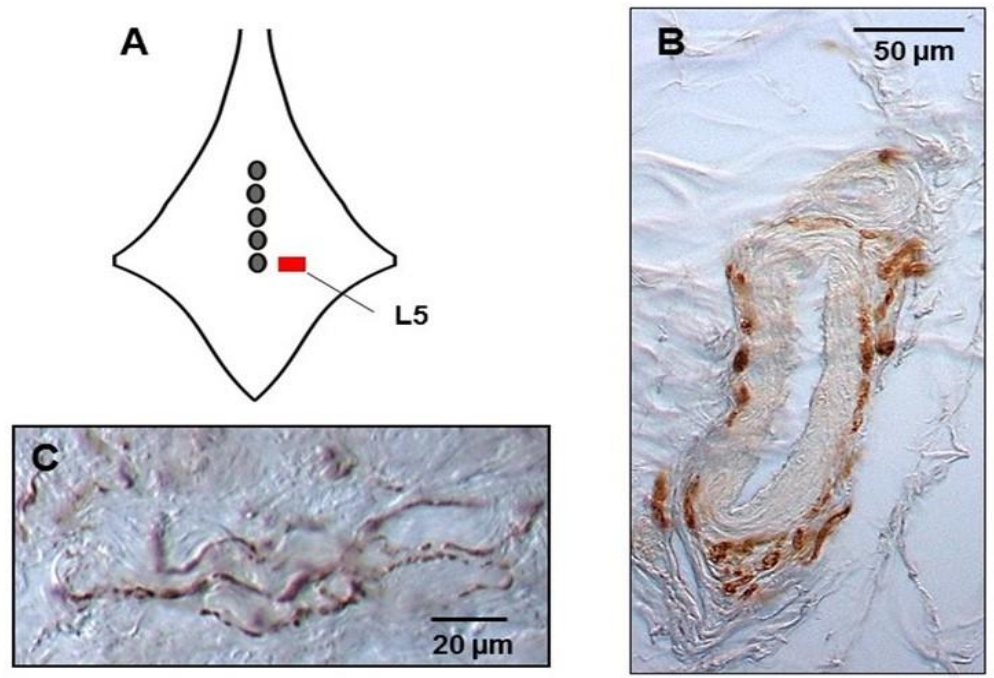

Fig 3. Cross-section of a human TLF stained with antibodies to PGP 9.5. A, dorsal view of a scheme of the TLF showing the site close to vertebra L5 (red quadrangle) where the biopsy specimen were taken. B, nerve ending with many varicosities in the adventitia of a small artery. $C$, free nerve ending with several chains of varicosities in the inner layer.

shows a SP-containing free nerve ending in the outer layer of the rat TLF.

\section{Sympathetic efferent fibres and endings}

The enzyme tyrosine hydroxylase (TH) is involved in the synthesis of the catecholamines dopamine, adrenaline und noradrenaline. ${ }^{12}$ In peripheral nerves it occurs predominantly in postganglionic sympathetic fibres, where it can be detected by antibodies to $\mathrm{TH}$. The main action of TH-ir fibres is vasoconstriction, and most of the TH-fibres in our histological material accompanied blood vessels. Fig. 6 shows a TH-ir ending in the wall of a small blood vessel. Because of its location, the ending is assumed to have a vasoconstrictive function. The density of the postganglionic sympathetic fibres was higher than that of SP-ir fibres, but lower than that of CGRP-ir ones

The effects of a fasciitis on the sensory innervation For quantitative evaluation of the fasciitis effects, only the units stained for PGP 9.5, CGRP, and SP were selected. An experimental fasciitis was induced by injection of Freund complete adjuvant into the fascia. Twelve days later the density of innervation for each fibre type was determined. The fasciitis was supposed to not only mimic the symptoms of a clinical fasciitis but

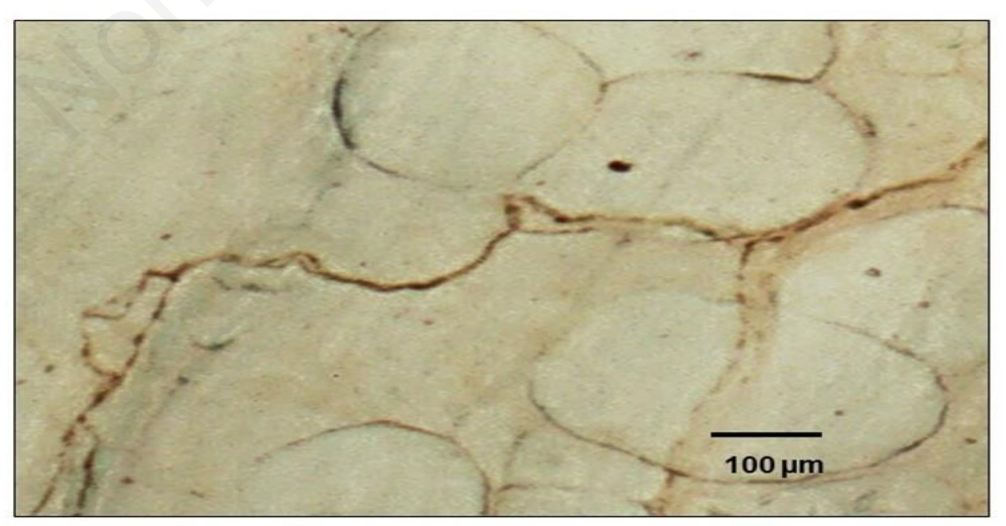

Fig 4. Whole mount preparation of the rat TLF stained with antibodies to CGRP. A network of small nerve fibres is visible that accompany blood vessels that are barely visible (in the right lower corner). Some fibres to the left exhibit varicosities. 


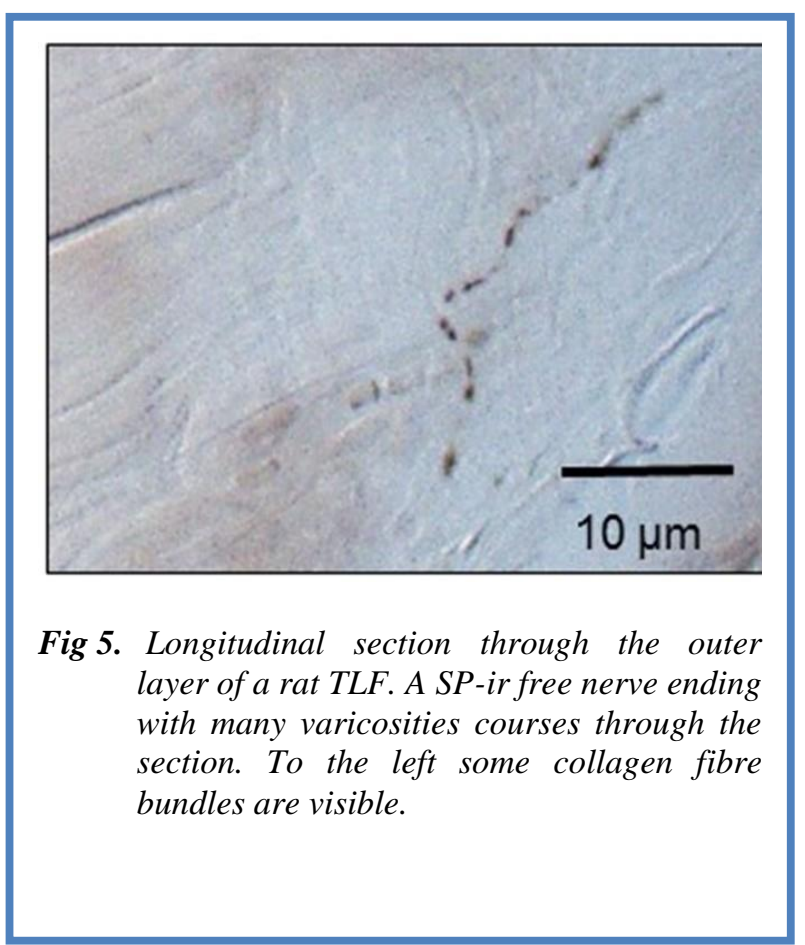

also of other long-lasting painful alterations of the fascia. The histological sections showed a localized swelling at the injection site and massive infiltrations of leukocytes in all layers of the fascia. At high magnifications eosinophilic granulocytes and lymphoid cells were visible. The histological picture resembled an eosinophilic inflammation. ${ }^{13}$ There was no difference in the morphology between free nerve endings in the inflamed and intact fascia. The inflamed fascia exhibited a higher density of CGRP-ir and SP-ir units (Figure 7B,C). However, the density of PGP 9.5containing units - which represented all fibres and endings in the fascia - was not higher (Figure 7A). This apparent discrepancy is due to the fact that the TH-ir sympathetic units had a much lower density in the inflamed fascia (not shown). The reason for this decrease in TH-ir fiber density is not known. Schaible and Straub have likewise described a changed sympathetic supply to joints when they were experimentally inflamed. ${ }^{14}$ The inflammation-induced changes in innervation density occurred in the subcutaneous tissue and the inner layer; they were almost absent in the middle layer. Moreover, we never found a SP-ir fibre in this layer, and this was valid for the intact and inflamed fascia. The mechanism through which the fibre length increases in inflamed tissue is obscure. The two main possibilities are sprouting or increased branching of the fibers. In a study on afferents from the intact and inflamed gastrocnemius-soleus muscle, also NGF-ir and growth associated protein 43[(GAP 43-)] ir fibers were increased. ${ }^{15}$ This finding points to sprouting as the most important mechanism. So, an increased density of nociceptive fibers in inflamed tissue appears to be a general phenomenon.

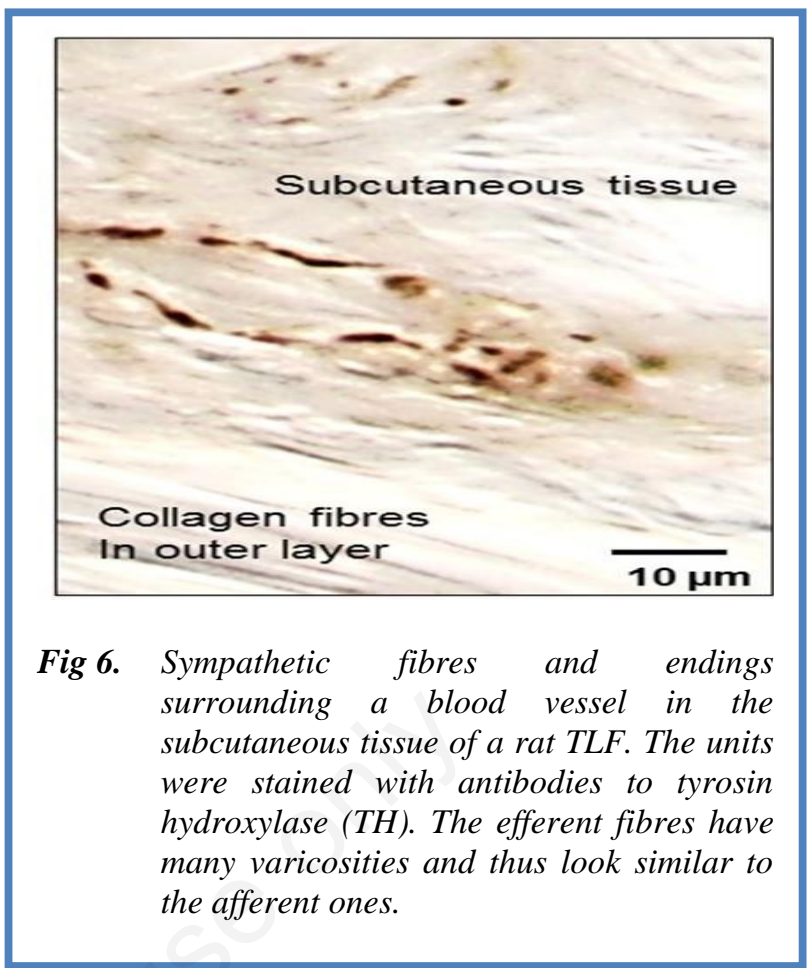

\section{Discussion}

According to our data, the TLF is not elastic in the sense of "stretchy". The only layer that contained a few elastic fibres was the inner layer. Since this layer consists of loose connective tissue, elastic forces cannot build up in the inner layer. However, after a deformation of the fascia, reversibility is possible because of the lattice grille-like arrangement of the collagen bundles in the middle layer, as described for the human fascia. ${ }^{16}$ The oblique collagen fibre bundles in the middle layer can slide against each other when the trunk is bent, and they can slide back again. The single collagen fibre bundle is probably relatively stiff, but their spatial arrangement could provide a certain resilience. For full reversibility, however, the flat abdominal muscles are probably needed.

\section{Proprioception}

Generally, the main function ascribed to the TLF is proprioception. The fact that we did not find one of the typical proprioceptors (muscle spindles, Ruffini corpuscles, Golgi organs) is surprising since other authors have described such endings in the intraspinal ligaments and human TLF. ${ }^{17,18}$ We looked closely with various staining techniques for such endings in the rat and human fascia, but without success. One explanation for this difference between the literature and our findings is that the other authors studied parts of the TLF we did not look at. All our data were obtained from a small piece of fascia lateral to the spinous processes. Therefore, it is possible that the TLF contains proprioceptors in more lateral areas of the TLF. Actually, not all parts of a fascia and not all fasciae are 


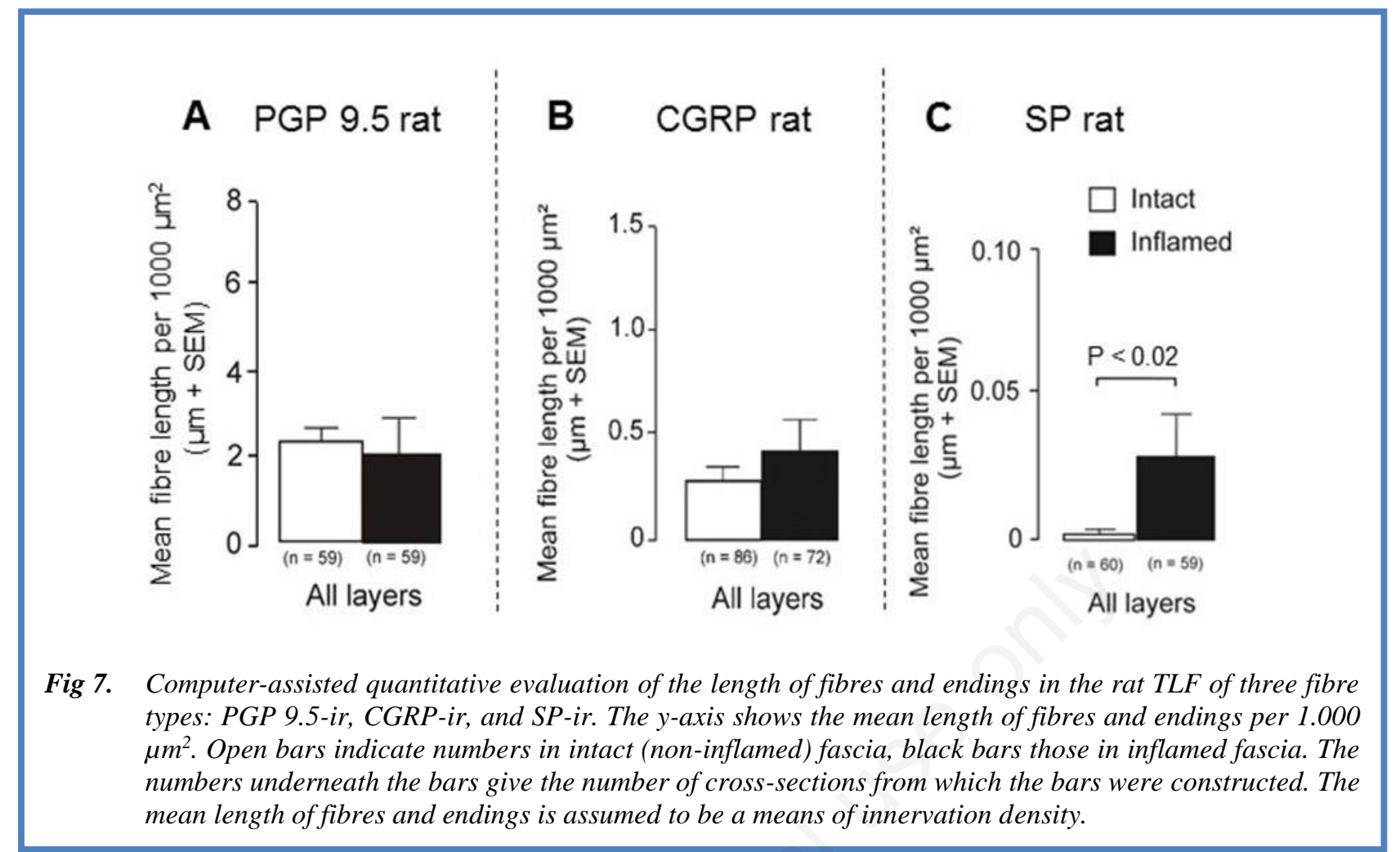

equipped with the same innervation. ${ }^{18}$ Therefore, the lack of proprioceptors in our tissue samples does not mean that the TLF has no proprioceptive function. Besides the fact that in other parts of the fascia proprioceptors may exist, the low-threshold mechanosensitive free nerve endings with group III and IV afferents found in muscle and diaphragm are probably also present in the TLF and - because of their low mechanical threshold - could function as proprioceptors. ${ }^{9,19}$

\section{Nociception}

Several lines of evidence point to the TLF as a source of pain.

\section{Nociceptor level}

The presence of SP in a free nerve endings has been shown to be associated with a nociceptive nature of the receptors. ${ }^{11}$ Our finding that the SP-containing endings were the only ones that exhibited a significant increase in density in inflamed TLF fits in the above observation (Figure 7). The data by Lawson and colleagues were obtained from the skin in guinea pigs, ${ }^{11}$ not from fascia. There is just one study in which the receptive properties of free nerve endings in the crural fascia were determined together with their neuropeptide content. ${ }^{20}$ All units tested in the study of Taguchi and colleagues were classified as nociceptors and contained CGRP in their axons. ${ }^{20}$ However, among the CGRP-positive endings many non-nociceptive receptors appear to exist besides nociceptive ones. ${ }^{21}$ In the present study, most of the SP- and CGRP-ir nerve endings probably fulfilled a nociceptive function. Moreover, we also found TRPV1ir endings that are assumed to be the main receptor molecules in the membrane of nociceptors. ${ }^{22}$

Spinal cord level

Previous studies of our group have shown that the mechanoreceptors and nociceptors of the TLF have an effective connection to the wide-dynamic range (WDR) neurons in the dorsal horn of the spinal cord. The WDR neurons are generally assumed to be the most common nociceptive neurons in the dorsal horn. We did not find neurons with exclusive input from the TLF; all the neurons recorded from had convergent input also from the skin and other soft tissues of the low back. ${ }^{23}$ Apparently, the fascia nociceptors are the beginning of a nociceptive pathway from the soft tissues of the low back to the spinal dorsal horn and probably further to higher centres. Since the input from all soft tissues of the low back converges on the same dorsal horn neurons, no separate pathway from fascia to higher centres appears to exist. This may be one reason why pain from the TLF is difficult to distinguish from the pain from other soft tissues of the low back.

Clinical level

The thoracolumbar fascia is involved in many painful disorders of the soft tissues in the low back. Examples besides fasciitis - are delayed onset muscle soreness and chronic low back pain to name a few. ${ }^{24,25}$ Injections of pain-producing substances into the TLF of human volunteers have shown that the fascia is the most painful 


\section{Innervation of the thoracolumbar fascia}

Eur J Transl Myol 29 (3): 151-158, 2019

soft tissue of the low back, more painful than skin, subcutaneous tissue and muscle. ${ }^{26}$

\section{Postganglionic sympathetic fibres}

The great proportion of sympathetic fibres in our specimen was surprising. A semiquantitative calculation showed that approximately $40 \%$ of the entire fascia innervation consisted of postganglionic sympathetic fibres. Probably, the majority of these fibres are vasoconstrictors, ${ }^{27}$ because for the other sympathetic efferents (e.g. sudomotor, pilomotor, cardiomotor, secretomotor $^{28}$ ) there are no targets in the fascia proper. In the subcutaneous tissue, however, many blood vessels exist that are innervated by sympathetic fibres (Figure 6). Since under psychologic stress the sympathetic activity is higher, the dense sympathetic innervation may explain why many patients with low back pain report increased pain levels when they are under stress.

In conclusion, the tissue specimen studied were taken from the TLF close to the spinous processes in rat and human. In this part of the TLF, only free nerve endings were found. None of the typical proprioceptors (muscle spindles, Golgi tendon organs, Ruffini corpuscles) existed in the specimen. This does not mean that TLF has no proprioceptive function, because many of the free nerve endings are sensitive to proprioceptive stimuli. The positive staining with antibodies to SP and TRPV1 showed that the TLF has an extensive innervation with nociceptors and therefore could constitute a pain source for low back pain.

\section{List of acronyms}

DAB - 3,3-diaminobenzidine tetrahydrochloride

CGRP - calcitonin gene-related peptide

GAP 4 - growth associated protein 43

PGP 9.5 - Protein gene product 9.5

SP - substance P

TH - tyrosine hydroxylase

TLF - thoracolumbar fascia

TRPV1 - transient receptor potential cation channel subfamily $\mathrm{V}$ member 1

WDR - wide-dynamic range

\section{Acknowledgments}

The valuable technical assistance help by E. Hofmann is gratefully acknowledged. Part of the data have been presented at the Fascia Symposium at Coburg (Germany) on June $10^{\text {th }}, 2017$.

\section{Funding}

The project is part of the research Consortium LOGIN (Localized and Generalized Musculoskeletal Pain: Psychobiological Mechanisms and Implications for Treatment) funded by the German Federal Ministry of Education and Research (01EC1010B)

\section{Conflict of Interest}

The author has no conflict of interest to declare.

\section{Ethical Publication Statement}

The author confirms that he read the Journal's position on issues involved in ethical publication and affirm that this report is consistent with those guidelines.

\section{Corresponding Author}

Siegfried Mense, Prof. Dr. med., Dreibüchelackerstr.13, D-69198 Schriesheim, Germany. Phone: 0049-6220-914 974; e-mail: siegfried.mense@ medma.uni-heidelberg.de

\section{References}

1. Stecco C, Adstrum S, Hedley G, et al. Update on fascial nomenclature. J Bodyw Mov Ther 2018;22:354. doi: 10.1016/j.jbmt.2017.12.015.

2. Stecco C, Sfriso MM, Porzionato A, et al. Microscopic anatomy of the visceral fasciae. J Anat 2017;231:121-8. doi: 10.1111/joa.12617.

3. Gatt A; Zito PM. Anatomy, Skin, Fascias: StatPearls 2018, Oct. 27

4. Tesarz J, Hoheisel U, Wiedenhofer B, Mense S. Sensory innervation of the thoracolumbar fascia in rats and humans. Neuroscience 2014;194, 302-8.

5. Bednar DA, Orr FW, Simon GT. Observations on the pathomorphology of the thoracolumbar fascia in chronic mechanical back pain. A microscopic study. Spine 1995;20:1161-4.

6. Yahia L, Rhalmi S, Newman N, Isler M. Sensory innervation of human thoracolumbar fascia: An immunohistochemical study. Acta Orthop Scand 1992;63:195-7.

7. Zimmermann M. Ethical guidelines for investigations of experimental pain in conscious animals. Pain 1983;16:109-10.

8. Stacey MJ. Free nerve endings in skeletal muscle of the cat. J Anat 1969;105:231-54.

9. Hoheisel U, Unger T, Mense S. Excitatory and modulatory effects of inflammatory cytokines and neurotrophins on mechanosensitive group IV muscle afferents in the rat. Pain 2005;114:168-76.

10 Levine JD, Fields HL, Basbaum AI. Peptides and the primary afferent nociceptor. J Neurosci 1993;13:2273-2286.

11. Lawson SN, Crepps BA, Perl ER. Relationship of substance $P$ to afferent characteristics of dorsal root ganglion neurones in guinea-pig. J Physiol 1997;505:177-91.

12. Nagatsu T. Tyrosine hydroxylase: human isoforms, structure and regulation in physiology and pathology. Essays in Biochemistry 1995;30:15-35.

13. Lebeaux D, Sène D. Eosinophilic fasciitis (Shulman disease). Best Pract Res Clin Rheumatol 2012;26:449-58.

14. Schaible HG, Straub RH. Function of the sympathetic supply in acute and chronic experimental joint inflammation. Auton Neurosci 2014;182:55-64. doi: 0.1016/j.autneu.2013.12.004. 


\section{Innervation of the thoracolumbar fascia}

Eur J Transl Myol 29 (3): 151-158, 2019

15. Reinert A, Kaske A, Mense S. Inflammationinduced increase in the density of neuropeptideimmunoreactive nerve endings in rat skeletal muscle. Exp Brain Res 1998;121:174-80.

16. Willard FH, Vleeming A, Schuenke MD, et al. The thoracolumbar fascia: anatomy, function and clinical considerations. J Anat 2012;221:507-36. doi: 10.1111/j.1469-7580.2012.01511.

17. Jiang H, Russell G, Raso VJ et al. The nature and distribution of the innervation of human supraspinal and interspinal ligaments. Spine 1995;20:869-76.

18. Stecco C, Gagey O, Belloni A et al. Anatomy of the deep fascia of the upper limb. Second part: study of innervation. Morphologie 2007;91:38-43.

19. Bałkowiec A, Kukuła K, Szulczyk P. Functional classification of afferent phrenic nerve fibres and diaphragmatic receptors in cats. J Physiol 1995;483:759-68.

20. Taguchi T, Yasui M, Kubo A et al. Nociception originating from the crural fascia in rats. Pain 2013; 154:1103-14. doi: 10.1016/j.pain.2013.03.017.

21. Kruger L, Silverman JD, Mantyh PW et al. Peripheral patterns of calcitonin-gene-related peptide general somatic sensory innervation: cutaneous and deep terminations. J Comp Neurol 1989;280:291-302.

22. Caterina MJ, Schumacher MA, Tominaga $\mathrm{M}$ et al. The capsaicin receptor: a heat-activated ion channel in the pain pathway. Nature 1997;389:816-24.

23. Hoheisel $U$, Mense $S$. Inflammation of the thoracolumbar fascia excites and sensitises rat dorsal horn neurons. Eur J Pain 2015;19:419-28.

24. Gibson W, Arendt-Nielsen L, Taguchi $\mathrm{T}$ et al. Increased pain from muscle fascia following eccentric exercise: animal and human findings. Exp Brain Res 2009;194:299-308.

25. Langevin HM, Sherman KJ. Pathophysiological model for chronic low back pain integrating connective tissue and nervous system mechanisms. Med Hypotheses 2007;68:74-80.

26. Schilder A, Hoheisel U, Magerl W, et al. Sensory findings after stimulation of the thoracolumbar fascia with hypertonic saline suggest its contribution to low back pain. Pain 2014;155:22231.

27. Yuan Sheng and Li Zhu. The crosstalk between autonomic nervous system and blood vessels. Int $\mathbf{J}$ Physiol Pathophysiol Pharmacol 2018;10:17-28.

28. Jänig W, Häbler HJ. Neurophysiological analysis of target-related sympathetic pathways--from animal to human: similarities and differences. Acta Physiol Scand 2003;177:255-74.

Submission: May 20, 2019

Revision received: June 26, 2019

Acceptance: June 26, 2019 